\title{
External validation of a multivariable claims-based rule for predicting in-hospital mortality and 30-day post-pulmonary embolism complications
}

Craig I. Coleman ${ }^{1 *}$, W. Frank Peacock ${ }^{2}$, Gregory J. Fermann ${ }^{3}$, Concetta Crivera ${ }^{4}$, Erin R. Weeda ${ }^{1}$, Michael Hull ${ }^{5}$, Mary DuCharme ${ }^{5}$, Laura Becker ${ }^{5}$ and Jeff R. Schein ${ }^{4}$

\begin{abstract}
Background: Low-risk pulmonary embolism (PE) patients may be candidates for outpatient treatment or abbreviated hospital stay. There is a need for a claims-based prediction rule that payers/hospitals can use to risk stratify PE patients. We sought to validate the In-hospital Mortality for PulmonAry embolism using Claims daTa (IMPACT) prediction rule for in-hospital and 30-day outcomes.
\end{abstract}

Methods: We used the Optum Research Database from 1/2008-3/2015 and included adults hospitalized for PE (415. $1 \mathrm{x}$ in the primary position or secondary position when accompanied by a primary code for a PE complication) and having continuous medical and prescription coverage for $\geq 6$-months prior and 3-months post-inclusion or until death. In-hospital and 30-day mortality and 30-day complications (recurrent venous thromboembolism, rehospitalization or death) were assessed and prognostic accuracies of IMPACT with $95 \%$ confidence intervals (Cls) were calculated.

Results: In total, 47,531 PE patients were included. In-hospital and 30-day mortality occurred in 7.9 and $9.4 \%$ of patients and $20.8 \%$ experienced any complication within 30-days. Of the $19.5 \%$ of patients classified as low-risk by IMPACT, $2.0 \%$ died in-hospital, resulting in a sensitivity and specificity of $95.2 \%$ (95\% Cl, 94.4-95.8) and $20.7 \%$ (95\% Cl, 20.4-21.1). Only 1 additional low-risk patient died within 30-days of admission and $12.2 \%$ experienced a complication, translating into a sensitivity and specificity of $95.9 \%(95 \% \mathrm{Cl}, 95.3-96.5)$ and $21.1 \%(95 \% \mathrm{Cl}$, 20.7-21.5) for mortality and $88.5 \%(95 \% \mathrm{Cl}, 87.9-89.2)$ and $21.6 \%(95 \% \mathrm{Cl}, 21.2-22.0)$ for any complication.

Conclusion: IMPACT had acceptable sensitivity for predicting in-hospital and 30-day mortality or complications and may be valuable for retrospective risk stratification of PE patients.

Keywords: Pulmonary embolism, Risk stratification, Mortality, Administrative claims

\section{Background}

Acute pulmonary embolism (PE) is the most serious clinical presentation of venous thromboembolic disease and has an incidence in United States (US) of 112 events per 100,000 individuals [1]. PE results in a substantial economic burden, with annual healthcare costs per case ranging from $\$ 13,018$ to $\$ 16,644$ [2].

\footnotetext{
* Correspondence: craig.coleman@hhchealth.org

${ }^{1}$ University of Connecticut School of Pharmacy, 69 North Eagleville Road,

Storrs, CT 06269, USA

Full list of author information is available at the end of the article
}

According to US and European PE treatment guidelines $[3,4]$, PE patients deemed at low-risk of experiencing early post-PE complications (including mortality) and who have adequate home circumstances should be considered candidates for treatment at home or following an abbreviated hospital admission. While clinical rules for the risk stratification of patients with $\mathrm{PE}$ are available [5], their implementation requires access to vital sign and laboratory data often incompletely reported or not found in claims databases. 
Providing researchers, payers and hospital administrators with a tool that allows them to retrospectively estimate PE patients' predicted early complication risk may facilitate epidemiologic research and aid them in making future resource utilization more efficient. The In-hospital Mortality for PulmonAry embolism using Claims daTa (IMPACT) prediction rule was derived [6] and subsequently validated in multiple external administrative databases for this purpose $[7,8]$ and has shown prognostic accuracy for predicting in-hospital mortality similar to that of the PE severity index (PESI), simplified PESI (sPESI) and Hestia criteria. However, sparse data supporting IMPACT's ability to predict 30-day post-PE mortality and other complications are available [9]. Here, we sought to externally validate IMPACT's accuracy for predicting in-hospital and 30-day outcomes using administrative claims data contained in the Optum Research Database.

\section{Methods}

The preparation of this research report was in accordance with the Transparent Reporting of a multivariable prediction model for Individual Prognosis Or Diagnosis (TRIPOD) statement [10].

Our analysis used claims data from the Optum Research Database spanning January 2008 through March 2015. The Optum Research Database contains de-identified claims data from commercial and Medicare Advantage health plan patients and links administrative enrollment data with medical (physician, facility) and pharmacy claims [11]. Since this study utilized only deidentified patient level data via methods consistent with Health Insurance Portability and Accountability Act (HIPAA) privacy and security requirements (i.e., individual medical records or identities were not disclosed), institutional review board oversight was not required.

We included adult patients with an International Classification of Diseases, ninth-edition, Clinical Modification (ICD-9-CM) diagnosis code for PE (415.1x) in the primary position or with a secondary diagnosis code for PE along with a primary code for a PE-related complications (respiratory failure [518.81], cardiogenic shock [785.51], cardiac arrest [427.5], secondary pulmonary hypertension [416.8], syncope [780.2], thrombolysis [99.10] or intubation/mechanical ventilation [96.04, 96.05, 96.70-96.72]). Additional inclusion criteria consisted of continuous medical and prescription coverage for $\geq 6$-months prior and 3-months post-study discharge or until death. Patients transferred from another healthcare facility were excluded as determination of low-risk status by clinicians is typically performed at the time of initial PE assessment. In addition, including transfer patients would have biased our estimates of hospital length-of-stay.
We used the IMPACT prediction rule [estimated percent absolute risk $=1 /(1+\exp (-\mathrm{x}))$; where $\mathrm{x}=-5.833+(0.026 \times$ age $)+(0.402 \times$ myocardial infarction $)+(0.368 \times$ chronic lung disease $)+(0.464 \times$ stroke $)+(0.638 \times$ prior major bleeding $)+(0.298 \times$ atrial fibrillation $)+(1.061 \times$ cognitive impairment $)+(0.554 \times$ heart failure $)+(0.364 \times$ renal failure $)$ $+(0.484 \times$ liver disease $)+(0.523 \times$ coagulopathy $)+(1.068 \times$ cancer)] to estimate PE patients' risk for early all-cause mortality [6]. This claims-based logistic regression prediction tool was initially derived and internally validated in a large US MarketScan commercial and Medicare claims database by randomly assigning PE admissions between April 2010 and September 2013 into derivation (80 \%) and validation $(20 \%)$ cohorts. In both cohorts, the model classified PE patients into low- and high-risk in-hospital allcause mortality categories with high sensitivity (87\%) and moderate specificity (47\%). Consistent with prior studies, patients with an IMPACT predicted mortality risk $\leq 1.5 \%$ were classified as low-risk for early mortality or other complications in our analysis [6-9]. ICD-9-CM coding for all IMPACT co-morbidities was performed according to the original IMPACT derivation paper [6]. Whenever possible, these co-morbidities were determined using Agency for Healthcare Research and Quality (AHRQ) 29-comorbidity schemas [12]; however, prior major bleeding, cognitive dysfunction, stroke, myocardial infarction and atrial fibrillation are not included in the AHRQ 29- comorbidity score and were thus identified using the procedural and diagnostic codes as listed in Additional file 1. Age was determined at time of presentation.

All-cause in-hospital and 30-day mortality as well as 30-day incidence of post-PE complications (recurrent venous thromboembolism, rehospitalization or death) served as a priori endpoints for this study. In-hospital and 30-day mortality were determined using the discharge status field within the claims records and from using the Social Security Administration Death Master File. Rehospitalization was said to occur if a patient had a new all-cause inpatient claim anytime following discharge for the index PE event through 30-days postadmission for the index event. Recurrent venous thromboembolism was defined as a diagnosis code for PE or deep vein thrombosis (see Additional file 2) on an emergency department or inpatient claim within 30-days of the index event. Accordingly, patients not discharged within 30-days of the index admission were not included in measures of 30-day post-admission rehospitalization and recurrent venous thromboembolism.

All baseline variables and endpoints were analyzed descriptively. Counts and percentages were provided for dichotomous or categorical variables. Means \pm standard deviations or medians with $25 \%, 75 \%$ ranges were provided for continuous variables (where appropriate). To quantify the accuracy of IMPACT for predicting in- 
hospital and 30-day mortality as well as 30-day post-PE complications, we calculated sensitivity (the percentage of patients at high risk for a complication who are correctly identified as being high risk as evidenced by a complication occurring), specificity (the percentage of patients at low-risk of a complication who are correctly identified as being low-risk as evidenced by not experiencing a complication), positive predictive value (PPV; the probability that in the case of being classified as high-risk for a complication, the patient experiences a complication) and negative predictive value (NPV; the probability that in the case of being classified as low-risk for a complication, the patient does not experience one) along with $95 \%$ confidence intervals (CIs). Area underthe-curve (AUC) statistics were calculated to assess the IMPACT rule's discriminative power to correctly predict complication occurrence. All data management and statistical analysis was performed using SAS version 9.4 (SAS Institute Inc, Cary, North Carolina, USA).

\section{Results}

In total, 47,531 PE patients were identified (Table 1). The mean patient age was $67.1 \pm 15.0$ years, with $63.2 \%$ of patients $\geq 65$ years-of-age. A majority of patients (63.3 \%) were enrolled in Medicare Advantage plans rather than commercial insurance. The most common IMPACT co-morbidities observed were chronic lung disease (34.2\%), heart failure (23.1\%), atrial fibrillation $(16.4 \%)$ and cancer $(15.3 \%)$. High-risk patients were considerably older (mean age 72.1 versus 46.6 years, $p<0.001$ ) and had a higher prevalence of total comorbidities than the low-risk patients. The mean IMPACT score was estimated to be $6.1 \% \pm 8.1 \%$ for the total population,

Table 1 Baseline characteristics for low- and high-risk patients

\begin{tabular}{llll}
\hline Characteristic & Total Cohort & $\begin{array}{l}\text { IMPACT Low-Risk } \\
N=47,531\end{array}$ & $\begin{array}{l}\text { High-Risk } \\
N=9,259\end{array}$ \\
& $n(\%)$ & $n(\%)$ & $n=38,272$ \\
& $67.1 \pm 15.0$ & $46.6 \pm 11.1$ & $72.1 \pm 11.0$ \\
\hline Age, years & & & \\
(mean \pm SD) & $1,647(3.5)$ & $14(0.2)$ & $1,633(4.3)$ \\
Myocardial infarction & $16,242(34.2)$ & $636(6.9)$ & $15,606(40.8)$ \\
Chronic lung disease & $15(0.2)$ & $2,162(5.6)$ \\
Stroke & $2,177(4.6)$ & $101(1.1)$ & $6,132(16.0)$ \\
Prior major bleeding & $6,233(13.1)$ & $51(0.6)$ & $7,759(20.3)$ \\
Atrial fibrillation & $7,810(16.4)$ & $3(<0.1)$ & $6,486(16.9)$ \\
Cognitive dysfunction & $6,489(13.7)$ & $50(0.5)$ & $10,917(28.5)$ \\
Heart failure & $10,967(23.1)$ & $60(0.7)$ & $6,881(18.0)$ \\
Renal failure & $6,941(14.6)$ & $38(0.4)$ & $1,405(3.7)$ \\
Liver disease & $1,443(3.0)$ & $75(0.8)$ & $2,753(7.2)$ \\
Coagulopathy & $2,828(6.0)$ & $4(<0.1)$ & $7,283(19.0)$ \\
Cancer & $7,287(15.3)$ & &
\end{tabular}

$S D$ standard deviation and differed significantly between patients at low-risk $(1.1 \% \pm 0.3 \%)$ or high-risk $(7.3 \% \pm 8.6 \%)$ for early mortality or complications $(p<0.001)$.

In-hospital and 30-day mortality occurred in 7.9 and $9.4 \%$ of patients. The observed mortality risk for patients increased as estimated IMPACT mortality risk increased (see Additional file 3). A total of $20.8 \%$ of patients experienced any post-PE complication within 30-days of the index admission. Two-by-two cross-tables of events for each endpoint are provided in Table 2. Of the $19.5 \%$ of patients classified as low-risk by IMPACT, $2.0 \%$ died in-hospital (versus $9.4 \%$ in the high risk group, $p<0.001$ ), resulting in a sensitivity and specificity of 95.2 and $20.7 \%$ and a NPV of $98.0 \%$ (Table 3). Only 1 additional low-risk patient died within 30-days of admission and $12.2 \%$ experienced a complication, translating into a sensitivity, specificity and NPV of 95.9, 21.1 and $98.0 \%$ for mortality and 88.5, 21.6 and $87.8 \%$ for any complication. IMPACT's AUCs for the in-hospital mortality, 30-day mortality and 30-day complication endpoints were $0.66,0.68$ and 0.62 , respectively.

Median hospital length-of-stay was 4-days (25\%, $75 \%$ range: $2-6$ days) in the low-risk group and 5-days (25\%, $75 \%$ range: $3-9$ days) in the high-risk group. The percentage of patients with inpatient stays $\leq 2$-days was $32.1 \%$ (2975 of 9259) in the low-risk group and $20.5 \%$ $(7853$ of 38,272$)$ in the high-risk group $(p<0.001)$.

\section{Discussion}

The multivariable IMPACT prediction rule appeared valid when applied retrospectively to the Optum Research Database. In this and prior external validation studies [6-9], IMPACT has exhibited sensitivity $>90 \%$ and NPVs $\geq 98 \%$ for all-cause in-hospital mortality, but often with lower and variable specificity. While a prognostic rule would ideally be $100 \%$ sensitive and $100 \%$ specific; this is rarely seen in real-world scenarios. However because first and foremost clinicians aim to avoid doing harm to their patients, high sensitivity is clearly preferable (and low specificity is less important) when assessing whether a PE patient could have (claims-based tools) or should have (clinical tools) been considered for management at home or following an abbreviated hospital stay (e.g., observation status).

In this study, we also assessed 30-day all-cause mortality, and found IMPACT to have similar sensitivity and NPV (95.9 and $98.0 \%$, respectively) to clinical tools such as PESI, sPESI and the Hestia criteria [5]. These results support those of a smaller $(N=807)$ single-site study of computed tomography-confirmed PE patients published by Weeda and colleagues [9] that compared IMPACT's predictive accuracy for 30-day all-cause mortality to that of PESI, sPESI and Hestia. In the study by Weeda et al. [9], IMPACT demonstrated comparable sensitivity and 
Table $22 \times 2$ Tables for All-Cause In-Hospital and 30-Day Mortality and 30-Day Complications

\begin{tabular}{|c|c|c|}
\hline & $\begin{array}{l}\text { No All-Cause In-Hospital } \\
\text { Death }\end{array}$ & $\begin{array}{l}\text { All-Cause In-Hospital } \\
\text { Death }\end{array}$ \\
\hline IMPACT High-Risk & 34,687 & 3,585 \\
\hline \multirow[t]{2}{*}{ IMPACT Low-Risk ${ }^{\mathrm{a}}$} & 9,077 & 182 \\
\hline & $\begin{array}{l}\text { No 30-Day } \\
\text { All-Cause Death }\end{array}$ & $\begin{array}{l}\text { 30-Day } \\
\text { All-Cause Death }\end{array}$ \\
\hline IMPACT High-Risk & 34,013 & 4,259 \\
\hline \multirow[t]{2}{*}{ IMPACT Low-Risk ${ }^{a}$} & 9,076 & 183 \\
\hline & $\begin{array}{l}\text { No 30-Day Recurrent VTE, } \\
\text { Rehospitalization } \\
\text { or Death From } \\
\text { Any Cause }\end{array}$ & $\begin{array}{l}\text { 30-Day Recurrent VTE, } \\
\text { Rehospitalization } \\
\text { or Death From } \\
\text { Any Cause }\end{array}$ \\
\hline IMPACT High-Risk & 29,531 & 8,741 \\
\hline IMPACT Low-Risk ${ }^{a}$ & 8,130 & 1,129 \\
\hline
\end{tabular}

IMPACT In-hospital Mortality for PulmonAry embolism using Claims daTa, VTE venous thromboembolism

aLow-risk defined as an "In-hospital Mortality for PulmonAry embolism using

Claims daTa" rule estimated risk of early complications of $\leq 1.5 \%$

NPV for all-cause 30-day mortality (sensitivity $=97.0 \%$; $\mathrm{NPV}=99.4 \%$ ) compared to PESI, sPESI and Hestia (30day mortality sensitivity range $=90.9-100 \%$ and NPV range $=98.6-100 \%)$. This similar sensitivity is not surprising as IMPACT contains variables (including age, cancer, altered mental status, heart failure and chronic lung disease) found in commonly cited clinical rules (e.g., sPESI and/or PESI). Further, the magnitude/weight assigned to these overlapping predictors is similar across IMAPCT and clinical tools (e.g., altered mental status and cancer are heavily weighted in IMPACT and PESI). Unfortunately, we could not directly compare the prognostic accuracy of IMPACT to that of clinical rules in our analysis. While the Optum Research Database does have an electronic health record component allowing them to link vital signs and laboratory results to a proportion of covered patients, initial a priori pilot analyses performed in preparation for this study suggested too limited data were available to support the measurement of PESI, sPESI or the Hestia criteria. For this reason, and because IMPACT was specifically designed to risk stratify $P E$ patients retrospectively using administrative claims data, we strongly encourage it not be used to make individual patient treatment decisions. Instead, we believe the value of IMPACT lies in its ability to retrospectively identify low-risk PE patients, making it a excellent tool for payers and hospital administrators to quickly and inexpensively benchmark rates of low-risk PE patients treated at home or following an abbreviated admission.

Of note, this study was the first to evaluate the accuracy of IMPACT for stratifying patient risk for developing a complication (recurrent venous thromboembolism, rehospitalization or death) within 30-days. IMPACT's sensitivity for this endpoint was found to be $88.5 \%$; somewhat lower than observed for either in-hospital or 30-day mortality, but still likely acceptable for many decision-makers. This unique data demonstrating IMPACT's prognostic accuracy for 30-day post-PE complications is important as various payors such as the Centers for Medicare and Medicaid Services (CMS) continue to put pressure on providers to reduced the rate of hospital readmissions [13].

Our study has other limitations worth discussing. First, while claims data are extremely valuable for the efficient and effective examination of real-world healthcare outcomes, treatment patterns, healthcare resource utilization and costs, all claims databases have inherent limitations affecting their internal and external validity. Results are dependent on the accuracy and completeness of administrative claims data, which by nature, may be prone to coding errors or omissions (for example, out of hospital mortality may have been missed due to poor reporting to the Social Security Administration and the need to link this data to Optum claims). Moreover, some limitation in the generalizability of results should always be considered because claims data are collected for the purpose of payment and not research. Second, we could not determine if the observed mortality or complications were attributable to the index PE, as this requires a prospective design and access to detailed chart data. It is likely some deaths or rehospitalizations were associated with co-morbidities (i.e., cancer and heart failure) and not directly related to the index PE. While it is unclear what influence this had on the sensitivity and specificity of IMPACT on our mortality and any complication endpoints, it may at least partially explain IMPACT's reduced sensitivity and NPV for the any complication (30day recurrent VTE, rehospitalization or death from any cause) endpoint. A final limitation includes the fact that claims data cannot provide information on severity of comorbidities (only their presence) and cannot fully address other factors that may be associated with the development of early complications (or the decision to keep patients in the hospital longer), including socioeconomic status, likely patient compliance to medical instructions and the presence of family support.

\section{Conclusion}

The multivariable IMPACT rule appeared valid for predicting early (up to 30-day) post-PE outcomes when implemented in the Optum Research Database. IMPACT has previously been shown to exhibit sensitivity of $\sim 90 \%$ and NPV 99 \% for predicting in-hospital and 30-day mortality; and in addition to confirming these findings, this study also provides data on IMPACT's ability to risk stratify patients for the development of recurrent venous thromboembolism, rehospitalization or 
Table 3 Prognostic Test Characteristics for the "In-hospital Mortality for PulmonAry embolism using Claims daTa" Rule

\begin{tabular}{|c|c|c|c|c|c|c|c|c|c|c|c|c|c|}
\hline & \multicolumn{2}{|l|}{ Impact } & \multicolumn{2}{|c|}{ Complication } & \multirow{3}{*}{$\begin{array}{l}\text { TP } \\
(\%)\end{array}$} & \multirow{3}{*}{$\begin{array}{l}\text { TN } \\
(\%)\end{array}$} & \multirow{3}{*}{$\begin{array}{l}\text { FP } \\
(\%)\end{array}$} & \multirow{3}{*}{$\begin{array}{l}\mathrm{FN} \\
(\%)\end{array}$} & \multirow{3}{*}{$\begin{array}{l}\text { Sensitivity } \\
(\mathrm{TP} / \mathrm{TP}+\mathrm{FN}) \\
(\%, 95 \% \mathrm{Cl})\end{array}$} & \multirow{3}{*}{$\begin{array}{l}\text { Specificity } \\
(\mathrm{TN} / \mathrm{TN}+\mathrm{FP}) \\
(\%, 95 \% \mathrm{Cl})\end{array}$} & \multirow{3}{*}{$\begin{array}{l}\text { NPV } \\
(\text { TN/TN + FN) } \\
(\%, 95 \% \mathrm{Cl})\end{array}$} & \multirow{3}{*}{$\begin{array}{l}\text { PPV } \\
(\mathrm{TP} / \mathrm{TP}+\mathrm{FP}) \\
(\%, 95 \% \mathrm{Cl})\end{array}$} & \multirow{3}{*}{$\begin{array}{l}\text { AUC } \\
(95 \% \mathrm{Cl})\end{array}$} \\
\hline & Low-risk & High-risk & No & Yes & & & & & & & & & \\
\hline & $N$ & $N$ & $N$ & $N$ & & & & & & & & & \\
\hline All-Cause In-Hospital Mortality & 9,259 & 38,272 & 43,764 & 3,762 & 9.4 & 98.0 & 90.6 & 2.0 & $95.2(94.4-95.8)$ & $20.7(20.4-21.1)$ & $98.0(97.7-98.3)$ & $9.4(9.1-9.7)$ & $0.66(0.65-0.67)$ \\
\hline All-Cause 30-Day Mortality & 9,259 & 38,272 & 43,089 & 4,442 & 11.1 & 98.0 & 88.9 & 2.0 & $95.9(95.3-96.5)$ & $21.1(20.7-21.5)$ & $98.0(97.7-98.3)$ & $11.1(10.8-11.5)$ & $0.68(0.67-0.69)$ \\
\hline $\begin{array}{l}\text { 30-Day Recurrent VTE, Rehospitalization or } \\
\text { Death From Any Cause }\end{array}$ & 9,259 & 38,272 & 37,661 & 9,870 & 22.8 & 87.8 & 77.1 & 12.2 & $88.5(87.9-89.2)$ & $21.6(21.2-22.0)$ & $87.8(87.1-88.5)$ & $22.8(22.4-23.3)$ & $0.62(0.62-0.63)$ \\
\hline
\end{tabular}

AUC area under the curve statistic, $C l$ confidence interval, $F N$ false negative, $F P$ false positive, IMPACT In-hospital Mortality for PulmonAry embolism using Claims daTa, $N$ number, $N P V$ negative predictive value, $P P V$ positive predictive value, $T N$ true negative, $T P$ true positive, VTE venous thromboembolism 
death at 30-days. While not intended for clinical decision-making, IMPACT may be a valuable tool for retrospective analysis or benchmarking of PE patients.

\section{Additional files}

Additional file 1: International Classification of Disease, Ninth RevisionClinical Modification (ICD-9-CM) codes for independent predictors in the IMPACT model. (DOCX $12 \mathrm{~kb})$

Additional file 2: Diagnosis Codes for Recurrent Venous Thromboembolism. (DOCX $13 \mathrm{~kb}$ )

Additional file 3: Calibration Plot of Observed In-hospital and 30-Day Mortality. (DOCX $353 \mathrm{~kb}$ )

\section{Abbreviations}

AHRQ: Agency for Healthcare Research and Quality; AUC: Area under-thecurve; Cls: Confidence intervals; CMS: Centers for Medicare and Medicaid Services; ICD-9-CM: International Classification of Diseases, ninth-edition, Clinical Modification; IMPACT: In-hospital Mortality for PulmonAry embolism using Claims daTa; NPV: Negative predictive value; PE: Pulmonary embolism; PESI: PE severity index; PPV: Positive predictive value; sPESI: simplified PESI; TRIPOD: Transparent Reporting of a multivariable prediction model for Individual Prognosis Or Diagnosis; US: United States

\section{Acknowledgements}

None.

\section{Funding}

This analysis was sponsored by Janssen Scientific Affairs, LLC, Raritan, NJ, USA.

\section{Availability of data and material}

The data supporting the conclusions of this article are included within the article. The detailed data set is not publicly available.

\section{Authors' contributions}

Study concept and design: CIC, WFP, GJF, CC, ERW, MH, MD, LB, JRS Acquisition of data: MH, MD, LB. Analysis and interpretation of data: CIC, WFP, GJF, CC, ERW, MH, MD, LB, JRS. Drafting the manuscript: CIC, WFP, GJF, CC, ERW, MH, MD, LB, JRS. Critical Revision of the manuscript for important intellectual content: CIC, WFP, GJF, CC, ERW, MH, MD, LB, JRS. Administrative, technical or material support: CIC. Study supervision: C.I.C. All authors read and approved the final manuscript.

\section{Authors' information}

Not applicable.

\section{Competing interests}

$\mathrm{CIC}$ has received grant funding and consultancy fees from Janssen Pharmaceuticals; Bayer Pharma AG and Boehringer-Ingelheim Pharmaceuticals, Inc. CC and JRS are employees of Janssen Scientific Affairs LLC. WFP has received grant funding and consultancy fees from Janssen Pharmaceuticals and Portola. GJF has received grant funding from Pfizer and is on the advisory board and speaker's bureau for Janssen Pharmaceuticals. MH, MD and LB are employees of Optum, Eden Prairie, MN, USA. ERW has no conflicts of interest germane to this manuscript to report.

\section{Consent for publication}

Not applicable.

\section{Ethics approval and consent to participate}

Since this study utilized only de-identified patient level data via methods consistent with Health Insurance Portability and Accountability Act (HIPAA) privacy and security requirements (i.e., individual medical records or identities were not disclosed), institutional review board oversight was not required. For this type of retrospective study, formal consent is not required.

\section{Author details}

'University of Connecticut School of Pharmacy, 69 North Eagleville Road, Storrs, CT 06269, USA. ²Department of Emergency Medicine, Baylor College of Medicine, Houston, TX, USA. ${ }^{3}$ Department of Emergency Medicine, University of Cincinnati, Cincinnati, OH, USA. ${ }^{4}$ Janssen Scientific Affairs, LLC, Raritan, NJ, USA. ${ }^{5}$ Optum, Eden Prairie, MN, USA.

Received: 15 July 2016 Accepted: 15 October 2016

Published online: 22 October 2016

\section{References}

1. Wiener RS, Schwartz LM, Woloshin S. Time trends in pulmonary embolism in the United States: evidence of overdiagnosis. Arch Intern Med. 2011;171: $831-7$.

2. Spyropoulos AC, Lin J. Direct medical costs of venous thromboembolism and subsequent hospital readmission rates: an administrative claims analysis from 30 managed care organizations. J Manag Care Pharm. 2007;13:475-86.

3. Kearon C, Akl EA, Ornelas J, Blaivas A, Jimenez D, Bounameaux H, et al. Antithrombotic Therapy for VTE Disease: CHEST Guideline and Expert Panel Report. Chest. 2016;149:315-52

4. Konstantinides SV, Torbicki A, Agnelli G, Danchin N, Fitzmaurice D, Galiè N, et al. 2014 ESC guidelines on the diagnosis and management of acute pulmonary embolism. Eur Heart J. 2014;35:3033-69.

5. Kohn CG, Mearns ES, Parker MW, Hernandez AV, Coleman CI. Prognostic accuracy of clinical prediction rules for early post-pulmonary embolism allcause mortality: A bivariate meta-analysis. Chest. 2015;147:1043-62.

6. Coleman Cl, Kohn CG, Bunz TJ. Derivation and validation of the In-hospital Mortality for PulmonAry embolism using Claims daTa (IMPACT) prediction rule. Curr Med Res Opin. 2015;31:1461-8.

7. Coleman Cl, Kohn CG, Crivera C, Schein JR, Peacock WF. Validation of the multivariable In-hospital Mortality for PulmonAry embolism using Claims daTa (IMPACT) prediction rule within an all-payer inpatient administrative claims database. BMJ Open. 2015;5:e009251.

8. Kohn CG, Peacock WF, Fermann GJ, Bunz TJ, Crivera C, Schein JR, et al. External validation of the In-hospital Mortality for PulmonAry embolism using Claims daTa (IMPACT) multivariable prediction rule. Int J Clin Pract. 2016;70:82-8.

9. Weeda ER, Kohn CG, Fermann GJ, Peacock WF, Tanner C, McGrath D, et al. External validation of prognostic rules for early post-pulmonary embolism mortality: assessment of a claims-based and three clinical-based approaches. Thromb J. 2016;14:7

10. Moons KG, Altman DG, Reitsma JB, loannidis JP, Macaskill P, Steyerberg EW, et al. Transparent Reporting of a multivariable prediction model for Individual Prognosis or Diagnosis (TRIPOD): explanation and elaboration. Ann Intern Med. 2015;162:W1-W73.

11. Optum. Retrospective database analysis. Optum Inc. 2013. Available at: https://www.optum.com/content/dam/optum/resources/productSheets/ Retrospective-Database-Analysis.pdf (Last accessed on 17 June 2016).

12. Elixhauser A, Steiner C, Harris DR, Coffey RM. Comorbidity measures for use with administrative data. Med Care. 1998;36:8-27.

13. Centers for Medicare and Medicaid Services. Hospital Readmissions Reduction Program (HRRP). Available at: https://www.cms.gov/medicare/ medicare-fee-for-service-payment/acuteinpatientpps/readmissionsreduction-program.html (Last accessed on 17 June, 2016). 